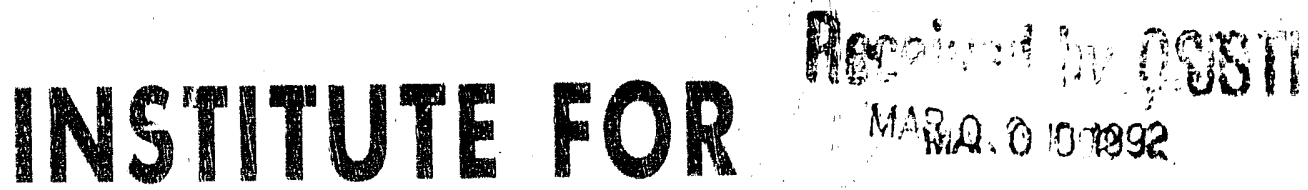 FUSION STUDIES
}

DOE/ET-53088-5333

IFSR \#533

On Zero Frequency Magnetic Fluctuations in Plasmas

T. TAJIMA and S. CABLE

Institute for Fusion Studies

The University of Texas at Austin

Austin, Texas 78712

and

R.M. KulsRUD

Department of Astrophysical Sciences

Princeton University

Princeton, N.J 05.540

January 1992

THE UNIVERSITY OF TEXAS

\section{AUSTIN}
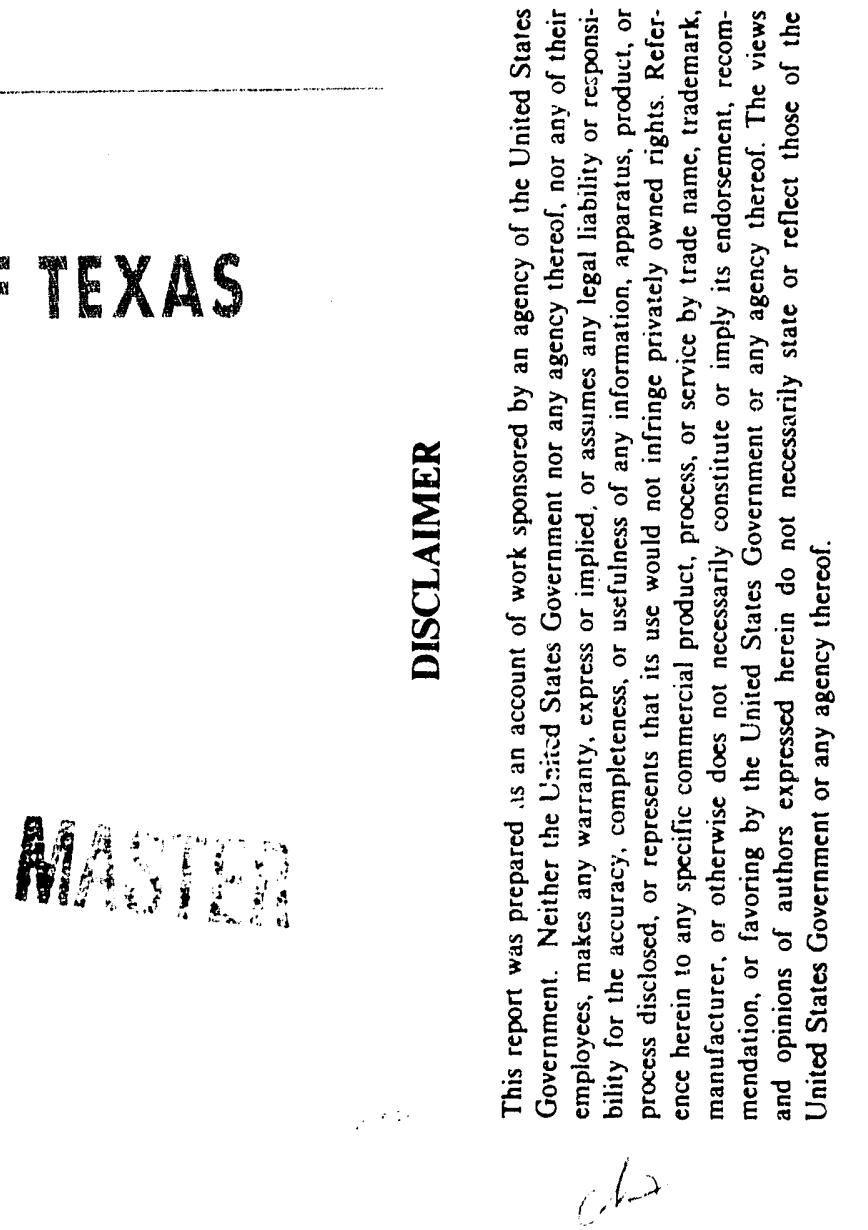


\title{
On Zero Frequency Magnetic Fluctuations in Plasmas
}

\author{
T. Tajima and S. Cable \\ Institute for Fusion Studies \\ The University of Texas at Austin \\ Austin, Texas 78712 \\ and \\ R.M. Kulsrud \\ Department of Astrophysical Sciences \\ Princeton University \\ Princeton, NJ 08540
}

\begin{abstract}
A plasma sustains fluctuations of electromagnetic fields and particle density even in a thermal equilibrium and such fluctuations have a large zero frequency peak. The level of fluctuations in the plasma for a given wavelength and frequency of electromagn tic fields is calculated through the fluctuation-dissipation theorem. The frequency sfectrum shows that the energy contained in this peak is complementary to the energy "lost" by the plasma cutoff effect. The level of the zero (or nearly zero) frequency magnetic fields is computed as $\left\langle B^{2}\right\rangle^{0} / 8 \pi=\frac{1}{2 \pi^{3}} T\left(\omega_{p} / c\right)^{3}$, where $T$ and $\omega_{p}$ are the temperature and plasma frequency. The relation between the nonradiative and radiative fluctuations is elucidated. Both a simple collision model and a kinetic theoretic treatment are presented with essentially the same results. The size of the fluctuations is $\lambda \sim\left(c / \omega_{p}\right)(\eta / \omega)^{1 / 2}$, where $\eta$ and $\omega$ are the collision frequency and the (nearly zero) frequency of magnetic fields nscillations. Perhaps the most dramatic application of the present theory, however, is to the cosmological plasma of early epoch (say
\end{abstract}


$t=10^{-2}-10^{0} \mathrm{sec}$ after the Big Bang). Implications of these magnetic fields in the early Universe are discussed. Quantum mechanical calculations are also carried out for degenerate plasmas. 


\section{Introduction}

Fluctuations of various physical quantities near zero frequency have been the active subject of research for a long time and a quite subtle one at least since Johnson ${ }^{1}$ and Nyquist ${ }^{2}$ discussed the subject. (One could begin citing even earlier works such as Einstein.) Some other early experimental works include Brophy and Rostoker ${ }^{3}$ and $\mathrm{McWhorter}{ }^{4}$ in semiconductors and some of the latest include papers by R. Stenzel ${ }^{5}$ and Zhang, DeSilva and Mostovych ${ }^{6}$ in plasmas. In $1957 \mathrm{Kubo}^{7}$ published a general theory on the fluctuation-dissipation theorcm. Rostoker et al. ${ }^{8,9}$ and Dawson et al. ${ }^{10,11}$ have theoretically looked at fluctuations in plasmas in a slightly different approach. In particular in Refs. 9 and 11 radiation from plasma has been considered in detail. Many of the previous authors on this subject were aware of the subtlety involved in low frequency fluctuations, including the work by Kadanoff and Martin, ${ }^{12}$ in which the relation to hydrodynamics was pointed out. A great deal of plasma works are compiled in Sitenko's book. ${ }^{13}$ To our best knowledge, however, we were unable to find a concrete expression of the low frequency spectrum of fluctuations of, say, magnetic fields in a thermal plasma. In the present paper, therefore, we investigate frequency spectra of magnetic fields and other physical quantities of a plasma in a thermal equilibrium near zero frequency.

Our investigation is based on the fluctuation-dissipation theorem. ${ }^{7}$ The derivation of the frequency spectrum of magnetic fluctuations in a nonmagnetized gaseous plasma is presented in Sec. II. We will find a peak around $\omega=0$ for magnetic fluctuations as well as for other physical quantities. The magnetic $\omega=0$ peak will be interpreted as the cvanescent energy component of electromagnetic fluctuations "screened" in a plasma below the plasma frequency. In Sec. III we briefly discuss kinetic and collisional effects on fluctuations. We will find that the lifetime (or the inverse of frequency) is related to the wavelength of fluctua- 
tions. Further, we will find that the amount of fuctuations of $\omega \approx 0$ is greater for hotter and denser plasmas. These findings lead to Sec. IV, in which the most spectacular application of the present theory turns out to be to the primordial cosmological plasma. We will describe some of the consequences of the present theory.

\section{The Fluctuation-Dissipation Theorem}

In or near thermal equilibrium the plasma has thermal fluctuations, whose level is related to the dissipative characteristics and the temperature $T$ of the plasma, as formulated in the fluctuation-dissipation theorem. ${ }^{7}$ We find an expression for the fluctuation spectrum of the magnetic field in an equilibrium plasma as a function of frequency. This is accomplished by deriving the magnetic fluctuations in wavenumber and frequency space $\left\langle B^{2}\right\rangle_{\mathrm{k} \omega} / \delta \pi$ from the fluctuation-dissipation theory, then integrating over wavenumber. $\left\langle B^{2}\right\rangle_{\omega} / 8 \pi$ is nearly a black-body spectrum at high frequencies, but, we are interested in low frequencies.

The following derivation closely parallels the work of Geary et al. ${ }^{14}$ We look at waves in a homogeneous isotropic nonmagnetized equilibrium plasma. We assume a wavevector $k=k \hat{x}$. The strength of electric field fluctuations may be found in Sitenko ${ }^{13}$ :

$$
\frac{1}{8 \pi}\left\langle E_{i} E_{j}\right\rangle_{\mathrm{k} \omega}=\frac{i}{2} \frac{\hbar}{e^{\hbar \omega / T}-1}\left\{\Lambda_{i j}^{-1}-\Lambda_{i j}^{-1^{*}}\right\}
$$

where

$$
\Lambda_{i j}(\omega, \mathrm{k})=\frac{c^{2} k^{2}}{\omega^{2}}\left(\frac{k_{i} k_{j}}{k^{2}}-\delta_{i j}\right)+\epsilon_{i j}(\omega, \mathrm{k}),
$$

where $\epsilon_{i j}(\omega, \mathbf{k})$ being the dielectric tensor of the plasma. Since Faraday's law is $\mathbf{B}=\frac{\mathrm{ck}}{\omega} \times \mathbf{E}$, and we have set $\mathrm{k}=k \widehat{x}$, we find

$$
\frac{\left\langle B_{2}^{2}\right\rangle_{\mathrm{k} \omega}}{8 \pi}=\frac{i}{2} \frac{\hbar}{e^{\hbar \omega / T}-1} \frac{c^{2} k^{2}}{\omega^{2}}\left\{\Lambda_{33}^{-1}-\Lambda_{33}^{-1^{*}}\right\}
$$

and 


$$
\frac{\left\langle B_{3}^{2}\right\rangle_{\mathrm{k} \omega}}{8 \pi}=\frac{i}{2} \frac{\hbar}{e^{\hbar \omega / T}-1} \frac{c^{2} k^{2}}{\omega^{2}}\left\{\Lambda_{22}^{-1}-\Lambda_{22}^{-1^{*}}\right\}
$$

where the subscript 1,2 , and 3 refer to $x, y$, and $z$. We then have the total magnetic fluctuations as

$$
\frac{\left\langle B_{\mathrm{tot}}^{2}\right\rangle_{\mathrm{k} \omega}}{8 \pi}=\frac{i}{2} \frac{\hbar}{e^{\hbar \omega / T}-1} \frac{c^{2} k^{2}}{\omega^{2}}\left\{\Lambda_{22}^{-1}+\Lambda_{33}^{-1}-\Lambda_{22}^{-1}{ }^{*}-\Lambda_{33}^{-1}{ }^{*}\right\}
$$

where c.c. refers to the complex conjugate.

In order to establish $\Lambda_{i j}(\omega, \mathrm{k})$, from the equation of motion of a plasma, here we introduce a multi-fluid model of a plasma. As a simple and analytically tractable model consider the case with finite and constant collisionality:

$$
m_{\alpha} \frac{d \mathbf{v}_{\alpha}}{d t}=e_{\alpha} \mathbf{E}-\eta_{\alpha} m_{\alpha} \mathbf{v}_{\alpha}
$$

where $\alpha$ is a particle species label and $\eta_{\alpha}$ is the collisional frequency but can include the viscosity effect. We can show that a description of electron dynamics more accurate than Eq. (6) such as kinetic treatments leads to better mathematical properties. We note that $\eta$ should tend to zero for very short wavelength EM waves because for large wavenumbers $k$ the photon shifts momentum by a large amount so that the interacting electron population before and after the intcraction becomes very small. However, for the sake of analytical tractability and physical transparency we take this simplified constant collision frequency model as illustration of the essential physics. Fourier transforming (6) gives

$$
-i \omega m_{\alpha} \mathrm{v}_{\alpha}=e_{\alpha} \mathbf{E}-\eta_{\alpha} m_{\alpha} \mathrm{v}_{\alpha}
$$

which yields the current $j_{\alpha}$

$$
\left(-i \omega+\eta_{\alpha}\right) \mathbf{j}_{\alpha}=\frac{\omega_{p \alpha}^{2}}{4 \pi} \mathbf{E}
$$

The susceptibility tensor $\chi_{\alpha i j}$ is deñned to relate $\mathrm{j}_{\alpha}$ to $\mathrm{E}$ such that

$$
j_{\alpha i}=-i \omega \chi_{\alpha i j}(\omega \mathrm{k}) E_{j}(\omega \mathrm{k})
$$


The dielectric tensor $e_{i j}(\omega \mathbf{k})$ is given by

$$
\epsilon_{i j}(\omega \mathrm{k})=\delta_{i j}+4 \pi \sum_{\alpha} \dot{\chi}_{\alpha i j}
$$

so

$$
4 \pi \chi_{\alpha i j}^{(\omega, \mathbf{k})}=\frac{\omega_{p \alpha}^{2}}{\omega\left(\omega+i \eta_{\alpha}\right)} \delta_{i j}
$$

and

$$
\epsilon_{i j}(\omega, \mathbf{k})=\delta_{i j}-\sum_{\alpha} \frac{\omega_{p \alpha}^{2}}{\omega\left(\omega+i \eta_{\alpha}\right)} \delta_{i j}
$$

In an electron-positron plasma (as we will apply the theory later to cosmology), we have $\omega_{p e^{+}}=\omega_{p e^{-}}$and $\eta_{e^{+}}=\eta_{e}=\eta$. Equation (12) becomes

$$
\epsilon_{i j}(\omega, \mathrm{k})=\delta_{i j}-\frac{\omega_{p}^{2}}{\omega(\omega+i \eta)} \delta_{i j}
$$

where $\omega_{p}^{2}=\omega_{p e^{+}}^{2}+\omega_{p e^{-}}^{2}$. We now obtain

$$
\Lambda_{i j}=\left(\begin{array}{l}
1-\frac{\omega_{p}^{2}}{\omega(\omega+i \eta)} \\
1-\frac{c^{2} k^{2}}{\omega^{2}}-\frac{\omega_{p}^{2}}{\omega(\omega+i \eta)} \\
1-\frac{c^{2} k^{2}}{\omega^{2}}-\frac{\omega^{2}}{\omega(\omega+i \eta)}
\end{array}\right)
$$

Combining Eqs. (5) and (14) after some algebra, we obtain

$$
\frac{\left\langle B^{2}\right\rangle_{\mathrm{k} \omega}}{8 \pi}=\frac{2 \hbar \omega}{e^{\hbar \omega / T}-1} \eta \omega_{p}^{2} \frac{k^{2} c^{2}}{\left(\omega^{2}+\eta^{2}\right) k^{4} c^{4}+2 \omega^{2}\left(\omega_{p}^{2}-\omega^{2}-\eta^{2}\right) k^{2} c^{2}+\left[\left(\omega^{2}-\omega_{p}^{2}\right)^{2}+\eta^{2} \omega^{2}\right] \omega^{2}} .
$$

Integrating Eq. (15) over wavenumber, we obtain

$$
\frac{\left\langle B^{2}\right\rangle_{\omega}}{8 \pi}=\frac{2 \hbar \omega}{e^{\hbar \omega / T}-1} \frac{2 \eta}{2 \pi^{2} \omega_{p e}^{2}}\left(\frac{\omega_{p e}}{c}\right)^{3} \int_{0}^{\infty} d x \frac{x^{4}}{\left(\omega^{\prime 2}+\eta^{\prime 2}\right) x^{4}+\cdots},
$$

where $x=k \frac{c}{\omega_{p e}}$ and the primed quantities are normalized by $\omega_{p e}$. Note that Eq. (16) diverges with $\eta^{\prime} \equiv \eta / \omega_{p e}$ fixed. 
The fact that we get high wavenumber divergence is not surprising, since we have, up to this point, based our calculations on classical fluid equations of motion with a constant collision frequency $\eta$ independent of $k$. We need a better theory to handle this difficulty. This may be done by a kinetic theory which includes more exact collision effects, waveparticle interaction, etc. However, by toing so, we overly clutter our expressions so that, we lose track of which effects give rise to zero frequency fluctuations and which do not. So, we proceed instead with this semi-phenomenological theory of collision effects in this section. However, we can show that both approaches yield essentially the same frequency spectra. In order to overcome the large $k$ divergence, we let $\eta$ tend to zero first, then we integrate over $k$ to infinity. This will bring the high frequency expression. This procedure physically corresponds to the vanishing cross-section of collisions as $k \rightarrow \infty$. We still need the correct plasma expression in the low frequency expression. At high frequency and high wavenumber $\left(\omega, c k \gg \omega_{p}\right)$ of Eq. (15), this function has a substantial value only where $\omega^{2}-c^{2} k^{2}-\omega_{p}^{2} \approx 0$. A high-frequency, high wavenumber limit is obtained by letting $\eta \rightarrow 0$. We take this limit with the aid of a standard definition of the Dirac $\delta$-function: Integrating Eq. (15) over $4 \pi k^{2} d k$ from 0 to $\infty$ with the above consideration, we obtain

$$
\frac{\left\langle B^{2}\right\rangle_{\omega}}{8 \pi}=\frac{T}{2 \pi} \delta(\omega) \int \frac{\omega_{p}^{2}}{\omega_{p}^{2}+c^{2} k^{2}} k^{2} d k+\frac{1}{2 \pi c^{3}} \frac{\hbar}{e^{\hbar \omega / T}-1}\left(\omega^{2}-\omega_{p}^{2}\right)^{3 / \omega} .
$$

This expression (17) reduces to the familiar black-body radiation formula in the limit $\omega_{p} \rightarrow 0$. As the $t_{1}$. "netic energy density is $\int_{-\infty}^{\infty} \frac{d \omega}{2 \pi} \frac{\left\langle B^{2}\right\rangle}{8 \pi}$ and half the cnergy in a black-body is stored in the electric field, we sce that this is the standard black-body spectrum. The only difference of the second term of Eq. (17) from the conventional vacuum formulation is the presence of the plasma cutoff $\omega_{p}$.

We now break up the integral of Eq. (16) into two intervals: one from zero to $h_{\text {cut }}$ and the other from $k_{\text {cut }}$ (or $x_{\text {cut }} \equiv k_{\text {cut }} c / \omega_{\text {pe }}$ ) to $\infty$. In the first integral we keep $\eta$ finite since 
the range of this integral includes $\omega \sim \mathcal{O}(\eta)$, while in the second integral we let $\eta \rightarrow 0$ :

$$
\begin{aligned}
\frac{\left\langle B^{2}\right\rangle_{\omega}}{8 \pi}= & \frac{1}{\pi^{2}} \frac{\hbar \omega^{\prime}}{e^{\left(\hbar \omega_{p e} / T\right) \omega^{\prime}}-1} 2 \eta^{\prime}\left(\frac{\omega_{p e}}{c}\right)^{3} \int_{0}^{x_{\mathrm{cut}}} d x \frac{x^{4}}{\left(\omega^{\prime 2}+\eta^{\prime 2}\right) x^{4}+\cdots} \\
& +\frac{\hbar\left(\omega^{\prime 2}-\omega_{p}^{\prime 2}\right)^{3 / 2}}{2 \pi\left(e^{\left(\hbar \omega_{p e} / T\right) \omega^{\prime}}-1\right)}\left(\frac{\omega_{p e}}{c}\right)^{3} \Theta\left(\omega-\sqrt{c^{2} k_{\mathrm{cut}}^{2}+\omega_{p}^{2}}\right)
\end{aligned}
$$

where $\Theta$ is the Heaviside step function. The second term is what we have obtained in the above. Note that the divergence of $\omega \rightarrow 0$ is removed:

$$
\lim _{\omega \rightarrow 0} \frac{\left\langle B^{2}\right\rangle_{\omega}}{\delta \pi}=\frac{\hbar \omega^{\prime}}{\pi^{2}\left(e^{\hbar \omega_{p e} \omega^{\prime} / T}-1\right)} 2\left(\frac{\omega_{p e}}{c}\right)^{3} \frac{1}{\eta^{\prime}} \int_{0}^{x_{c \mathrm{cut}}} d x=\frac{2 T}{\pi^{2} \eta^{\prime} \omega_{p e}}\left(\frac{\omega_{p e}}{c}\right)^{3} x_{\mathrm{cut}},
$$

where the second equality holds for the classical limit $T \gg \hbar \omega_{p e}$. We determine $k_{\text {cut }}$ (or $a_{\text {cut }}$ ) from the requirement that the frequency spectrum (18) be smooth at the joint between the low-frequency spectrum and the black-body spectrum. This gives a range of possible values of $k_{\text {cut }} . k_{\text {cut }}$ is fixed by satisfaction of equipartition law in the known limit. The size of $k_{\text {cut }}$ thus becomes approximately $k_{\text {cut }} \sim \omega_{\text {pe }} / c\left(x_{\text {cut }} \sim 1\right)$. This determines the spectrum intensity at $\omega \sim 0$. This intensity does not vary sensitively with $k_{\text {cut }}$. We show in Fig. 1 an example of the frequency spectrum of Eq. (18) of magnetic fluctuations. Near $\omega=0$ the spectrum goes like $\omega^{-2}$.

We now discuss the wavenumber spectrum. In the limit $\eta \rightarrow 0$ the integral of Eq. (15) over $\omega$ gives

$$
\frac{\left\langle B^{2}\right\rangle_{\mathbf{k}}}{8 \pi}=\frac{\hbar k^{2} c^{2}}{\left(e^{\hbar / T\left(\omega_{p}^{2}+k^{2} c^{2}\right)^{1 / 2}}-1\right)} \frac{1}{\left(\omega_{p}^{2}+c^{2} k^{2}\right)^{1 / 2}}+T \frac{\omega_{p}^{2}}{\omega_{p}^{2}+c^{2} k^{2}}
$$

First, note that, once again, $\omega_{p} \rightarrow 0$ gives the standard black-body result arising from the first term. (Remember: $\left\langle B^{2}\right\rangle / 8 \pi=\int_{0}^{\infty} \frac{d k}{(2 \pi)^{3}} 4 \pi k^{2}\left\langle B^{2}\right\rangle_{\mathrm{k}} / 8 \pi$.) The second term $i$ that given for $\left\langle B^{2}\right\rangle_{\mathrm{k}} / 8 \pi$ by Geary et al. ${ }^{14}$ They obtained this expression via the Darwin approximations. Our result satisfies both radiative and non-radiative limits. Note that when $\hbar\left(\omega_{p}^{2}+c^{2} k^{2}\right)^{1 / 2} \ll T$, two terms together yield

$$
\frac{\left\langle B^{2}\right\rangle_{k}}{8 \pi} \rightarrow T
$$


giving the equipartition law of classical statistical mechanics. We can show that this limit is obtained also by using the Kramers-Kronig theorem.

The integral over the zero frequency peak gives rise to the expression of the magnetic cnergy density near the zero frequency (i.e. non-radiative modes) as

$$
\frac{\left\langle B^{2}\right\rangle^{0}}{8 \pi}=\int_{0}^{\eta} \frac{d \omega}{2 \pi} \frac{\left\langle B^{2}\right\rangle_{\omega}^{0}}{8 \pi} \cong \int_{0}^{x_{\text {cul } \omega_{p} / c}} \frac{d k 4 \pi k^{2}}{(2 \pi)^{3}} \frac{\left\langle B^{2}\right\rangle_{\mathrm{k}}^{0}}{8 \pi}=\frac{1}{2 \pi^{3}} T\left(\frac{\omega_{p}}{c}\right)^{3} x_{\text {cint }},
$$

Another observation of interest may be made. The energy under the $\omega=0$ peak is approximately equal to the energy "deficit" around the plasma cutoff $\omega=\omega_{p}$. The cnergy under the peak is evaluated from $\mathrm{Eq} .(19)$ as $\sim T\left(\omega_{p} / c\right)^{3} 4 / \pi^{2}$. The energy deficit, using the Rayleigh-Jeans formula, is approximately

$$
\int_{-\omega_{p}}^{\omega_{p}} \frac{d \omega}{2 \pi} \frac{T}{2 \pi} \frac{\omega^{2}}{c^{3}}=\frac{4}{3 \pi^{2}} T\left(\frac{\omega_{p}}{c}\right)^{3} .
$$

This is pictorially shown in Fig. 2(a) with hatched areas with / and '. This result may be figuratively stated that the electromagnetic energy cutof by the plasma $\omega<\omega_{p e}$ is squeezed toward the zero frequency fluctuations when compared with the black-body radiation without plasma.

We also compare $\left\langle B^{2}\right\rangle_{\omega=0} / 8 \pi$ to the pressure produced by $\omega=0$ fluctuations in the ion density. Consider the longitudinal E-field fluctuations:

$$
\frac{\left\langle E_{x}^{2}\right\rangle}{8 \pi}=\frac{i}{2} \frac{\hbar}{e^{\hbar \omega / T}-1}\left(\Lambda_{11}^{-1}-\Lambda_{11}^{-1^{*}}\right)
$$

(Once again, $\mathrm{k}=k \hat{x}$.) The definition of $\Lambda_{i j}$ shows

$$
\Lambda_{11}=\epsilon_{11}(\omega, \mathrm{k}), \Lambda_{11}^{-1}=\frac{1}{\epsilon_{11}(\omega, \mathrm{k})} .
$$

By taking

$$
\epsilon_{11}(\omega, \mathrm{k})=1+\frac{k_{D}^{2}}{k^{2}}-\frac{\omega_{p i}^{2}}{\omega\left(\omega+i \eta_{i}\right)}
$$

we find

$$
\frac{\left\langle E_{x}^{2}\right\rangle_{k \omega}}{8 \pi}=\frac{\hbar \omega}{e^{\hbar \omega / T}-1} \frac{\eta \omega_{p i}^{2}}{\left[\omega^{2}\left(1+k_{D}^{2} / k^{2}\right)-\omega_{p i}^{2}\right]^{2}+\eta_{i}^{2} \omega^{2}\left(1+k_{D}^{2} / k^{2}\right)^{2}}
$$


where $k_{D}$ is the Debye wavenumber. Notice that while $\left\langle B^{2}\right\rangle_{\omega}$ is inversely proportional to $\eta$ at $\omega=0,\left\langle E_{x}^{2}\right\rangle_{\omega}$ is directly proportional to $\eta$ at $\omega=0$. Mathematically, this stems from the term $\frac{c^{2} k^{2}}{\omega^{2}}$ which is present in $\Lambda_{22}$ and $\Lambda_{33}$ (transverse fluctuations) but absent from $\Lambda_{11}$ (longitudinal).

Using the Poisson equation, we obtain the charge density fluctuations

$$
\left\langle\delta \rho^{2}\right\rangle_{\mathrm{k} \omega}=\frac{\hbar \omega}{\epsilon^{\hbar \omega / T}-1} \frac{k^{2} \eta \omega_{p i}^{2} / 2 \pi}{\left(\omega^{2}+\omega^{2} k_{D}^{2} / k^{2}-\omega_{p i}^{2}\right)^{2}+\eta^{2} \omega^{2}\left(1+k_{D}^{2} / k^{2}\right)^{2}}
$$

Now, this is total charge fluctuation. If we are interested in the ion density fluctuations, we use

$$
\delta n_{i}=\epsilon_{e} \delta \rho_{e} / e, \quad \text { where } \epsilon_{e}=1+\frac{k_{D}^{2}}{k^{2}}
$$

to obtain

$$
\left\langle\delta n_{i}^{2}\right\rangle_{\omega}=\frac{\hbar \omega}{e^{\hbar \omega / T}-1} \frac{\eta \omega_{p i}^{2}}{2 \pi e^{2}} \int_{0}^{k_{D}} \frac{d k}{(2 \pi)^{3}} 4 \pi k^{2}\left(1+\frac{k_{D}^{2}}{k^{2}}\right)^{2} \frac{k^{2}}{\left[\omega^{2}+\omega^{2} \frac{k_{D}^{2}}{k^{2}}-\omega_{p i}^{2}\right]^{2}+\eta^{2} \omega^{2}\left(1+\frac{k_{D}^{2}}{k^{2}}\right)^{2}} .
$$

In the zero frequency limit $\omega \rightarrow 0$ we have

$$
\left\langle\delta n_{i}^{2}\right\rangle_{\omega}=T \frac{\eta}{\omega_{p i}^{2}} \frac{1}{4 \pi^{3}} \frac{28}{15} k_{D}^{5} / e^{2}
$$

where $k_{D}$ is chosen as the cutoff since density fluctuations will be correlated very weakly for wavenumbers higher than $k_{D}$.

\section{Kinetic and Collisional Effects}

We find that the physical basis of the zero frequency peak is due to collisions (or other kinetic dissipation) or more precisely collision-induced quasi-modes. Imagine an individual charged particle, say, an electron propagates in a plasma, which itself is composed of an ensemble of such electrons (and other charged particles). With finite discreteness of the charge, the electron can contribute to a current fluctuation due to this ballistic motion of electron over the 
time until it encounters collisions (or other kinetic dissipation). Electromagnetic field fluctuations are induced from such a current fluctuation. However, the low frequency component of the field fluctuations finds itself that it cannot propagate, as it is evanescent, as distinct from the high frequency component which can propagate in a plasma as photon. Thus this gives rise to damping of the field fluctuations. Therefore, the lifetime of the fluctuations is related to the collision time (or other kinetic dissipation time).

The transverse part of the kinetic dielectric permitivity is given by

$$
\Lambda(\omega, \mathrm{k})=1-\frac{c^{2} k^{2}}{\omega^{2}}+\left(\frac{m}{2 \pi T}\right)^{1 / 2} \frac{\omega_{p e}^{2}}{\omega^{2}} \int_{L} \frac{e^{-m v^{2} / 2 T}}{-\omega+k v}+\left(\frac{M}{2 \pi T}\right)^{1 / 2} \int_{L} \frac{e^{-M v^{2} / 2 T}}{-\omega+k v}
$$

where $m$ is the electron mass, $M$ is the hydrogen ion mass, $\omega_{p e}$ is the electron plasma frequency, and $\omega_{p i}$ is the ion plasma frequency. The subscript " $L$ " attached to each integral sign is meant to specify the contour taken in each integral over the Landau contour. ${ }^{15}$

As indicated above, we are interested in fluctuations at frequencies and wave vectors in the regime

$$
\frac{\omega}{k} \leq v_{e}, v_{i}
$$

where $v_{e}=\sqrt{T / m}$ and $v_{i}=\sqrt{T / M}$. In this regime, we can approximate $\Lambda$ by

$$
\Lambda(\omega, \mathrm{k})=1-\frac{c^{2} k^{2}}{\omega^{2}}-\frac{\omega_{p e}^{2}}{v_{e}^{2} k^{2}}-\frac{\omega_{p i}^{2}}{v_{i}^{2} k^{2}}+i \sqrt{\frac{\pi}{2}} \frac{\omega_{p e}^{2}}{v_{e} \omega k}+i \sqrt{\frac{\pi}{2}} \frac{\omega_{p i}^{2}}{v_{i} \omega k} .
$$

Therefore, we obtain

$$
\frac{\left\langle B^{2}\right\rangle_{k \omega}}{8 \pi}=T \frac{2 \sqrt{\frac{\pi}{2}} \alpha \omega_{p e}^{2} c^{2} k^{5} / v_{e}}{\left(\omega^{2} k^{2}-c^{2} k^{4}-2 \omega_{p e}^{2} \omega^{2} / v_{e}^{2}\right)^{2}+\frac{\pi}{2} \alpha^{2} \omega_{p e}^{4} \omega^{2} k^{2} / v_{e}^{2}} .
$$

Here we see the qualitative confirmation of our earlier results: $\left\langle B^{2}\right\rangle_{\mathrm{k} w} / \delta \pi$ has a finite maximum at $\omega=0$ as long as $k^{2}<2 \omega_{p e}^{2} / v_{e}^{2}$. True enough, $\left\langle B^{2}\right\rangle_{\mathrm{k} \omega} / 8 \pi$ had a maximum at $\omega=0$ in the cold plasma, regardless of the size of $k$. In any event $\left\langle B^{2}\right\rangle_{\mathrm{k} \omega} / 8 \pi$ in the zerofrequency peak is on the order of $T / 2$ for small enough $k$. A rough approximation of the total energy density contained in this peak is then $T / 2$ times the $k$-space volume contained 
within the necessary wave-vector cutoff, divided by $(2 \pi)^{3}$. If we choose $k_{\text {cut }}=\omega_{p e} / c$ again, we find, once again,

$$
\frac{\left\langle B^{2}\right\rangle_{\mathbf{k} \omega}}{8 \pi}=\frac{T}{2} \frac{1}{6 \pi^{2}}\left(\frac{\omega_{p e}}{c}\right)^{3}
$$

From Maxwell's equations with all the terms on the right-hand side except the source term (the third term) written in terms of $\mathbf{E}$, we obtain the dispersion relation of the quasimodes:

$$
\omega^{2}-k^{2} c^{2}-\frac{\omega_{p}^{2}}{1+i \eta / \omega}=0 .
$$

In the low frequency limit Eq. (46) yields the dispersion relation

$$
\omega=i \frac{k^{2} c^{2}}{\omega_{p}^{2}} \eta
$$

Or, equivalently, the spatial size $\lambda$ of magnetic field fluctuations for a given lifetime $\tau_{l}\left(\equiv \omega^{-1}\right)$ is

$$
\lambda\left(\tau_{\ell}\right)=2 \pi \frac{c}{\omega_{p}}\left(\eta \tau_{\ell}\right)^{1 / 2} .
$$

Equation (36) states that the lifetime $\tau_{\ell}$ of magnetic fluctuations (or maybe called "magnetic bubble") of size $\lambda$ is proportional to the size squared $\left(T_{\ell} \propto \lambda^{2}\right)$; the larger the size of the bubble, the longer it lasts.

This entails an important ramification. Suppose two magnetic bubbles touch or collide with each other and coalesce into one. The time for coalescence of magnetic bubbles involves reconnection of magnetic field lines. It is generally known ${ }^{16,17}$ that this process (or related ones) is much faster than the diffusive time related to $\mathrm{Eq} .(36)$. Thus the coalescence time is much shorter than the individual life time. Therefore, before bubbles die away, they can form a coalesced bubble when they collide with each other, as long as they collide frequently enough. Once a larger coalesced bubble is formed, its life time is substantially longer, as Eq. (36) shows the life time is proportional to the square of the size of the bubble. It is possible to imagine that once larger bubbles are formed, they become even more long-lived 
and may be able to encounter more opportunities to collide with other bubbles. In this way a preferential formation of larger bubbles may become possible. This process is not far different from that of polynerization.

\section{Cosmological Significance}

We have found that the amount of zero-frequency magnetic fluctuations is proportional to $\mathrm{Tn}^{3 / 2}$ according to Eq. (22), where $n$ is the electron density. The higher the temperature and density, the greater the magnetic field fluctuations of the plasma become. Note that $T n^{3 / 2}=P n^{1 / 2}$ so that the zero-frequency magnetic fluctuations have stronger dependence on the plasma temperature than the pressure. This is why the most remarkable application of the present theory is found in the cosmological plasma. In fact during the radiation epoch of the cosmological time [i.e. $10^{-2}$ second after the Big. Bang till the $10^{13}$ second the main constituents of the Universe were photons, electrons (positrons) and ions (primarily protons)] the temperature $T$ of the photons and plasma is inversely proportional to the scale factor $a$ of the space time metric ${ }^{18}$ and $n \propto T^{3}$, so that $\left\langle B^{2}\right\rangle_{\omega=0} \propto a^{-11 / 2}$, while $P \propto a^{-4}$. Since $a(t) \propto \sqrt{t}$ during the radiation epoch, this means that the earlier the ephoch is, the greater the relative importance of the zero frequency magnetic field fluctuations to the plasma pressure is. In other words the plasma beta $\beta\left(=8 \pi P /\left\langle B^{2}\right\rangle_{\nu=0}\right) \propto a^{3 / 2}$ and around $t=10^{-2}$ second $\beta$ is nearly unity. At the very beginning of the radiation epoch (we may call it the plasma epoch as well) the present zero-frequency magnetic field fluctuations were most impressive, and we will start from then (prior to this epoch our knowledge on strong interaction is not sufficient enough to make any prediction). First, the present theory provides a mechanism for seed magnetic fields, which may help structure formation in the later Universe evolution. However, there are a few important questions. The typical spatial size of fluctuations is $c / \omega_{p}$, far, far smaller than any macroscopic cosmological scale. How can such small scales grow to cosmological macroscales? This is the question we will focus 
on in the rest of the present paper. In addition how large is the magnitude of magnetic fluctuations at the time of recombination $\left(t=10^{13} \mathrm{sec}\right)$ in the course of cosmic expansion? We defer this question to a future publication. Thirdly, can the magnetic fluctuations leave enough imprints on plasma density fluctuations for galaxy formation and at the same time not contradict with the observed highly uniform and isotropic cosmic microwave background? We will address this question briefly at the end of the present paper.

The process of successive coalescence (and its reverse) and decay of magnetic fluctuations in otherwise nonmagnetized plasmas is considered here. As our magnetic fluctuations have the lifetime greater for greater spatial sizes [Eq. (36)], we expect a "polymerization" of magnetic fluctuations (or bubbles) to occur. To illustrate this in a simplest possible way mathematically, we model this process as follows. We consider the magnetic fluctuations are ail composed of a typical size $a$ (which is about $c / \omega_{p}$ according to our findings). Our spatial scale herewith is normalized by this size $a$. When two such structures coalesce, its linear size becomes $i=2$. If three form a linear chain, $i=3$ etc. For the purpose of simplicity we consider only linear chains. There is a physical reason why the linear form is preferable for magnetic fluctuations, but detailed discussions on this are skipped here. Let $n_{i}$ be the number of chains of length $(i+1) a$. The lifetime of the elementary fluctuation according to Eq. (36) is $\tau_{0}=\lambda^{2} / \eta$. Then the lifetime of a chain with length $i a$ is $\tau_{0} i^{2}$. Since the coalescence time scale is much shorter than $\tau_{0}{ }^{17}$, the main time scale for the growth of the chain is determined by the "collision" frequency of bubbles. This is $\left\langle n_{0} v \sigma\right\rangle$, where $n_{0}$ is the number density of $i=1$ bubbles, $v$ is the typical velocity of the fluctuation, $\sigma$ the area (or "cross-section") of the bubbles. Then we obtain the following kinetic equation

$$
\frac{\partial n_{i}}{\partial t}=-\frac{n_{i}}{\tau_{0} i^{2}}+n_{i-1}\left\langle n_{0} v \sigma\right\rangle
$$

for all $i \geq 0$. We can add other coalescence processes such as $s$-th polymer with $n-s$ th polymer on the right-hand side of Eq. (38), i.e. $\Sigma \frac{1}{s^{2}} n_{i-s}\left\langle n_{s-1} v \sigma\right\rangle$ etc. For brevity sake, how- 
ever, we neglect these higher order processes herewith. In the expanding Universe Eq. (38) can be regarded as in the comoving coordinates. The physical quantities are related to the comoving quantities through

$$
\begin{aligned}
& n_{i}=n_{i}^{p} a^{3} \\
& \sigma=\sigma^{p} a^{-2} \\
& v=v^{p} a^{-1} \\
& \tau_{0}=\tau_{0}^{p} a^{2}
\end{aligned}
$$

so that the corresponding equation in physical space to Eq. (38) may be written as

$$
\frac{\partial}{\partial t} n_{i}^{p}+3 H n_{i}^{p}=-\frac{n_{i}^{p}}{\tau_{i}^{p} i^{2} a^{2}}+n_{i-1}^{p}\left\langle n_{0}^{p} v^{p} \sigma^{p}\right\rangle,
$$

where the superscript $p$ indicates physical space quantities. Note that $H \equiv \dot{a} / a$ and $\tau_{0}^{p}$ is (usually) assumed to be invariant if other quantities are fixed (i.e. the temperature etc.). Also note that the second term on the right-hand side is invariant. In the following we mainly treat Eq. (38) insteaud of Eq. (43).

We wish to solve the set of equations (38) under an appropriate boundary condition. In usual thermodynamics, thermodynamical equilibrium is understood as uniformly given elementary units. Thus we take $n_{0}(t)=n_{0}=$ const. By adopting non-dimensional units $n_{i} / n_{0} \rightarrow n_{i}$ and $t / \tau_{0} \rightarrow t$, we have

$$
\frac{\partial n_{i}}{\partial t}=-\frac{n_{i}}{i^{2}}+\kappa n_{i-1}
$$

where $\kappa \equiv \tau_{0}\left\langle n_{0} v \sigma\right\rangle$. In this section, for simplicity, we assume $\kappa$ is constant and much less than unity.

A stationary solution of $(44)$ as $t \rightarrow \infty$ is

$$
n_{i}=\kappa^{i}(i !)^{2} \simeq\left(\frac{\sqrt{\kappa} i}{e}\right)^{2 i} \text { for } i \gg 1 \text {. }
$$


Equation (45) says that in a stationary problem $(t \rightarrow \infty)$ there is always an infinite chain. Of course when an infinite chain begins to appear, we exhaust available elementary bubbles. This time $t_{*}$ and the size $i_{*}$ of bubbles at which the deprivation takes place are given as

$$
\begin{aligned}
& t_{*}=\tau_{0} \kappa^{-3 / 2} \\
& i_{*}=e \kappa^{-1 / 2} .
\end{aligned}
$$

Together with the initial and boundary conditions, we obtain ${ }^{19}$ the number of polymers of size $i$ at time $t$ as

$$
n(i, t)=\Theta[\kappa t-(i-1)] \exp \left[i-1+\frac{1}{\kappa}\right]\left(\frac{1}{i}-1\right)
$$

When we include the cosmic expansion ( $h \equiv \tau_{0} \dot{a} / a$, the normalized Hubble parameter), the growth time of the largest magnetic filament formation is greater than but on the order of $t_{*}$, which is calculated ${ }^{19}$ to be

$$
t_{*} \approx \frac{n}{\left(\frac{\partial n}{\partial i}\right)} \approx \frac{i *}{\kappa}=\sqrt{\frac{h^{1+b}}{k^{3}}} .
$$

Including the relaxation processes in a plasma, which are $h$ dependent in Eq. (49) and $h$, we obtain $^{19}$

$$
t_{*} \approx t_{1}\left(\frac{t}{t_{1}}\right)^{-\frac{1+b}{2}+\frac{3}{2}\left(\frac{9}{8}\right)} \approx t_{1}\left(\frac{t}{t_{1}}\right)^{-\frac{1+b}{2}+\frac{27}{16}},
$$

where $b$ is an index between 0 and 1 , depending on the relaxation process of the plasma, and $t_{1}$ is the beginning of the polymerization (we can assume $t_{1} \sim 10^{-2} \mathrm{sec}$ ). Equation (50) shows that $t_{*}=t_{1}\left(t / t_{1}\right)^{\alpha}$ where $\alpha \approx 2 / 3$, which implies that the horizon size magnetic strings are almost surely formed quickly within the Hubble time scale.

This implies, therefore, that during the radiation (i.e. plasma) epoch large spatial scale magnetic fluctuations can be formed, which could imprint their signatures on the plasma density. Zero-frequency magnetic fluctuations cause isothermal rather than adiabatic fluctuations in the plasma density. Therefore, the magnetic fluctuations can serve as a good 
candidate to carve out large structure formation in the plasma just prior to the recombination epoch with enough amplitude but without leaving any signature of inhomogeneity or anisotropy in the cosmic microwave background radiation (CMBR). This can be an explanation for what is believed ${ }^{20}$ to be the necessary density fluctuations $\delta n / n \sim 10^{-3}$ at the recombination epoch in order to generate present galaxies based on $N$-body computation and the observation constraint ${ }^{21}$ on CMBR of temperature fluctuations $\delta T / T<10^{-4}$. However, there remains a puzzle: Why the generated density fluctuation of $\delta r / n \sim 10^{-3}$ dces not create a corresponding photon red (or blue) shift in photon frequency in CMBR as required by the general theory of relatively ${ }^{18}$ ? This effect was first pointed out by Sachs and Wolfe. ${ }^{22}$

The preceding finding in the present paper, we believe, appears to give a possible rosolution to this problem. This is because the process of polymerization during the radiation epoch is so rapid that by the time of recombination large spatial structures are created in a time scale shorter than the photon diffusion time; the opaque plasma made the photon diffusion time over large structures nearly equal to the age of the Universe at that time. This implies that a photon which was originally emitted in a plasma prior to the density structure formation suffers the blue (or red) shift depending upon the sign of density perturbation, as the photon does not have enough time to escape out of this particular spatial spot. Upon the recombination the mean frce path for this photon becomes nearly infinite and thus will be. eventually observed by the terrestrial observer. When this photon travels out of the original density structure, the photon now has to climb up (or down) the gravitational potential so that it will acquire the exact same amount of frequency shift but this time it is a red (or blue) shift, correspondingly. As a result, the total frequency shift of this photon since the creation during the radiation epoch till the present day observation will be zero.

In conclusion, we have calculated the frequency spectra of fluctuations of magnetic fields and other physical quantities. We have found a sizeable zero-frequency peak in the magnetic frequency spectra. The amount of the magnetic fluctuation energy at $\omega \sim 0$ is proportional to 
the plasma temperature $T$ and the three halves power of the electron density $n$. The higher the temperature and density are, the greater the magnetic fluctuations. Thus the most dramatic implication of the present theory is on the cosmological primordial plasma. Intense magnetic field fluctuations of the order of $10^{16} \mathrm{G}$ may be expected at $t=10^{-2}$ sec after the Big Bang. Such magnetic fields may form larger spatial linkage through the process of successive coalescence (and its reverse). This physical process may be able to account for necessary cosmological constraints for large structure formation during the course of the evolution of the Universe. A great deal more work, however, is necessary in order to substantiate the above points as well as to explore other possible effects.

\section{Dedication}

Three days prior to the delivery of this paper at the American Physical Society meeting (i.e. Nov, 1, 1991), three of our friends and colleagues, Professor Dwight Nicholson, Professor Chris Goertz, and Professor Robert Smith, were innocently slain. We would like to dedicate this paper to the memory of these three colleagues, who dedicated their life to investigations of plasma physics and plasma astrophysics.

\section{Acknowledgments}

This work was supported by the U.S. Department of Energy and the National Science Foundation. We appreciate helpful discussions with Dr. K. Shibata, Dr. M. Isichenko, and Mr. S. Oliveira. 


\section{References}

1. J.B. Johnson, Phys. Rev. 32, 97 (1928).

2. H. Nyquist, Phys. Rev. 32, 110 (1928).

3. J.J. Brophy and N. Rostoker, Phys. Rev. 100, 754 (1955).

4. A.L. McWhorter, in "Semiconductor Surface Physics" ed. R.H. Kir.gston (Univ. Penn., Philadelphia, 1957) p. 207.

5. R. Stenzel, Phys. Fluids B 1, 1369 (1989).

6. Y.Z. Zhang, A.W. De Silva, and A.N. Mostovych, Phys. Rev. Lett. 62, 1848 (1989).

7. R. Kubo, J. Phys. S'oc. Jpn. 12, 570 (1957).

S. N. Rostoker and M.N. Rosenbluth, Phys. Fluids 3, 1 (1960).

9. N. Rostoker, R. Aamodt, and O. Eldridge, Ann. Phys. 31, 243 (1965).

10. J.M. Dawson and C. Oberman, Phys. Fluids 5, 517 (1962).

11. J.M. Dawson, in Advances in Plasma Physics eds. A. Simon and W.B. Thompson (Academic, New York, 1968) vol. 1, p. 1.

12. L.P. Kadanoff and P.C. Martin, Ann. Phys. 24, 419 (1963).

13. A.G. Sitenko, Electromagnetic Fluctuations in Plasma (Academic, New York, 1967).

14. J.L. Geary, T. Tajima, J.-N. Leboeuf, E.G. Zaidman, and J.H. Han, Comp. Phys. Comm. 42, 313 (1986).

15. L.D. Landau, Phys. Z. Sowjetunion 10,154 (1936). 
16. E.N. Parker, J. Geophys. Res, 62, 509 (1957).

17. T. Tajima and S.I. Sakai, Sov, J. Plasma Phys. 15, 606 (1989).

18. L.D. Landau and E.M. Lifshitz, The Classical Theory of Fields (Pergamon, Oxford, 1975); S. Weinberg, "Gravitation and Cosmology: Principles and Applications of the General Theory of Relativity" (Wiley, New York, 1972).

19. T. Tajima, S. Oliveira, and M. Isichenko, to be published.

20. for example, Ya.B. Zeldovich, Ap. Sp. Sci. Rev. 3, 1 (1984).

21. J.C. Mather, et al., Ap. J. Lett. 354, L37 (1990).

22. R.K. Sachs and A.M. Wolfe, Ap. J. 147, 73 (1967). 


\section{Figure Captions}

1. The frequency spectral intensity $S(\omega)=\left\langle B^{2}\right\rangle_{\omega} / 8 \pi$, e.g. plasma 1 sec after the Big Bang. $T=10^{100} \mathrm{~K} ; n_{\mathrm{e}}=4.8 \times 10^{30} / \mathrm{cc}$.

(a) $\ln \left(S(\omega) / S_{0}\right)$ plotted linearly in $\omega$. Zero-frequency peak is at the top of the g'aph, where $S_{0}$ is the normalization.

(b) $\ln \left(S(\omega) / S_{0}\right)$ plotted linearly in $\omega$. Zero-frequency peak is at the top of the graph, is seen to be higher than black-body peak.

(c) $\ln \left(S^{\prime}(\omega) / S_{0}\right)$ plotted logarithmically in $\omega$. Low-frequency line has slope around -2 . Rises to peak at $\omega=0$.

(d) $\ln \left(S(\omega) / S_{0}\right)$ plotted logarithmically in $\omega$. Note suppression of black-body radiation around $\omega=\omega_{p e}$.

2. Magnetic fluctuation spectra (a) Schematic plot of the spectrum of magnetic fluctuations $\left\langle B^{2}\right\rangle_{\omega} / 8 \pi$ in a thermal plasma with temperature $T$, plasma frequency $\omega_{p}$. The zero frequency peak has the height inversely proportional to the dissipation (such as collision frequency) and the width proportional to itself. The black-body profile is hardly modified. Only the low frequency $\left(\omega<\omega_{p}\right)$ is severely modified by the plasma

effects. (b) Schematic plot of the spectrum $\left\langle B^{2}\right\rangle_{k} / 8 \pi$. The shaded area corresponds to the second term in Eq. (20) and to the shaded area in Fig. 2(a). 

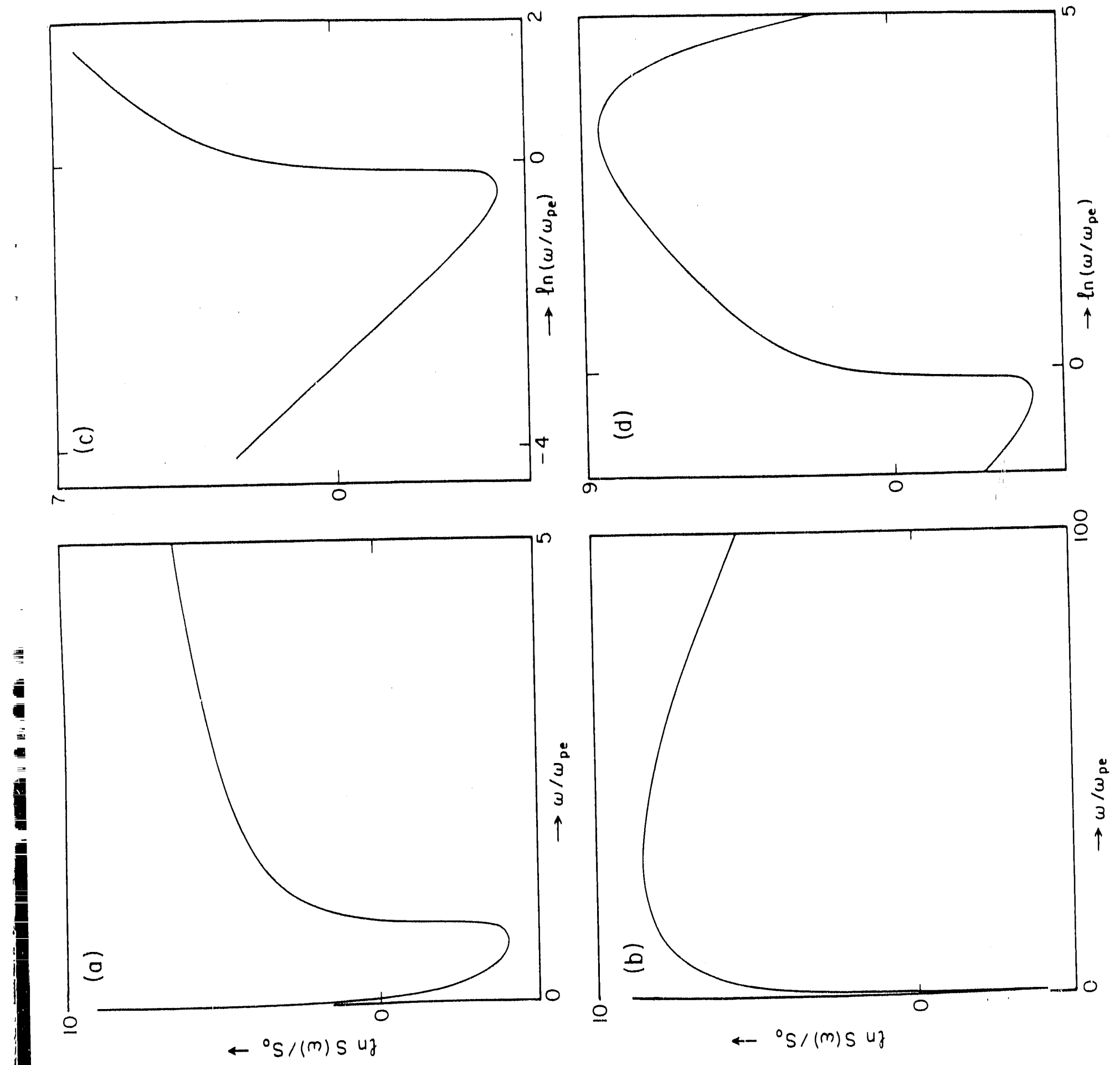

Fig. 1 

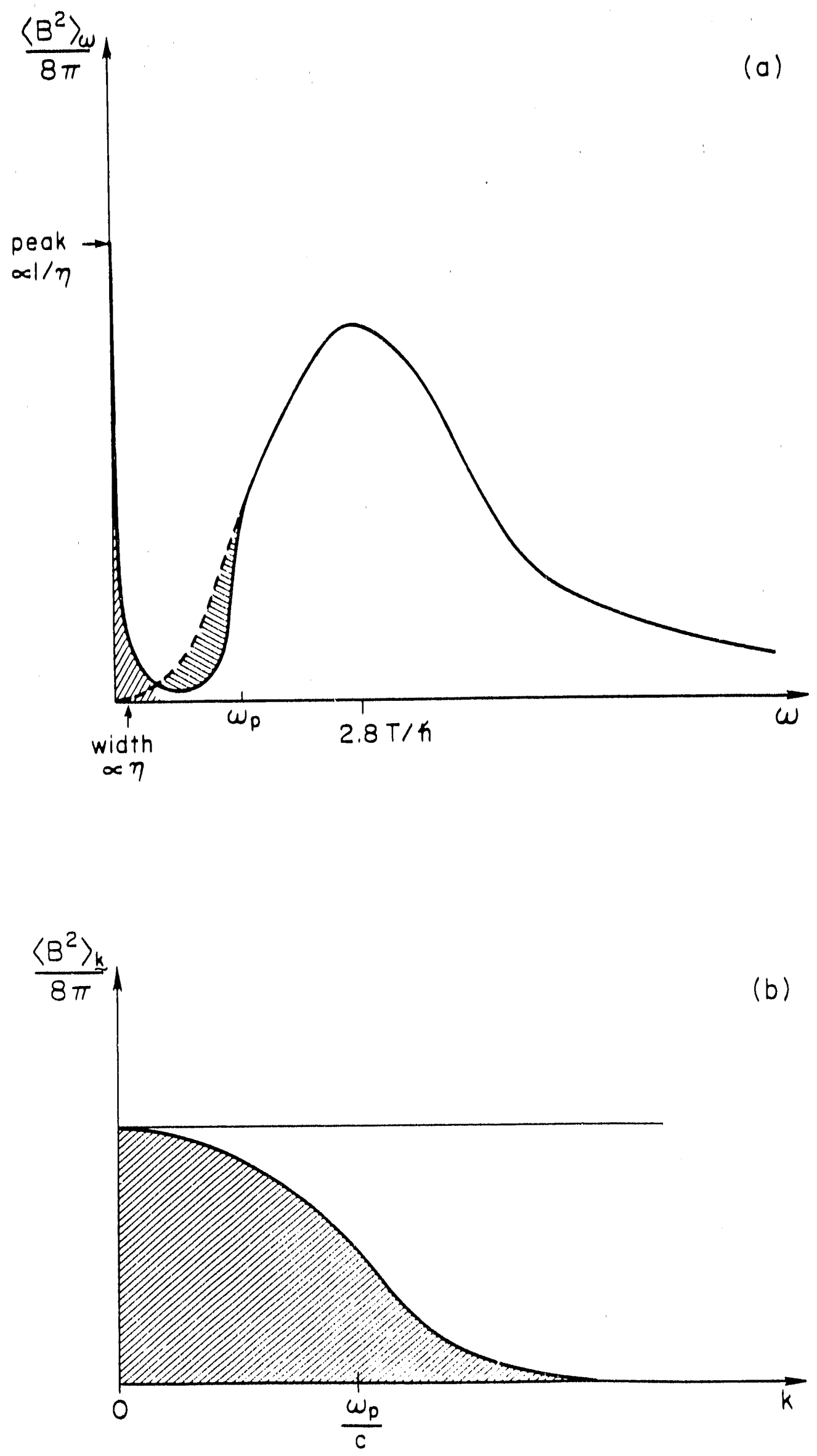

F1g. 2 

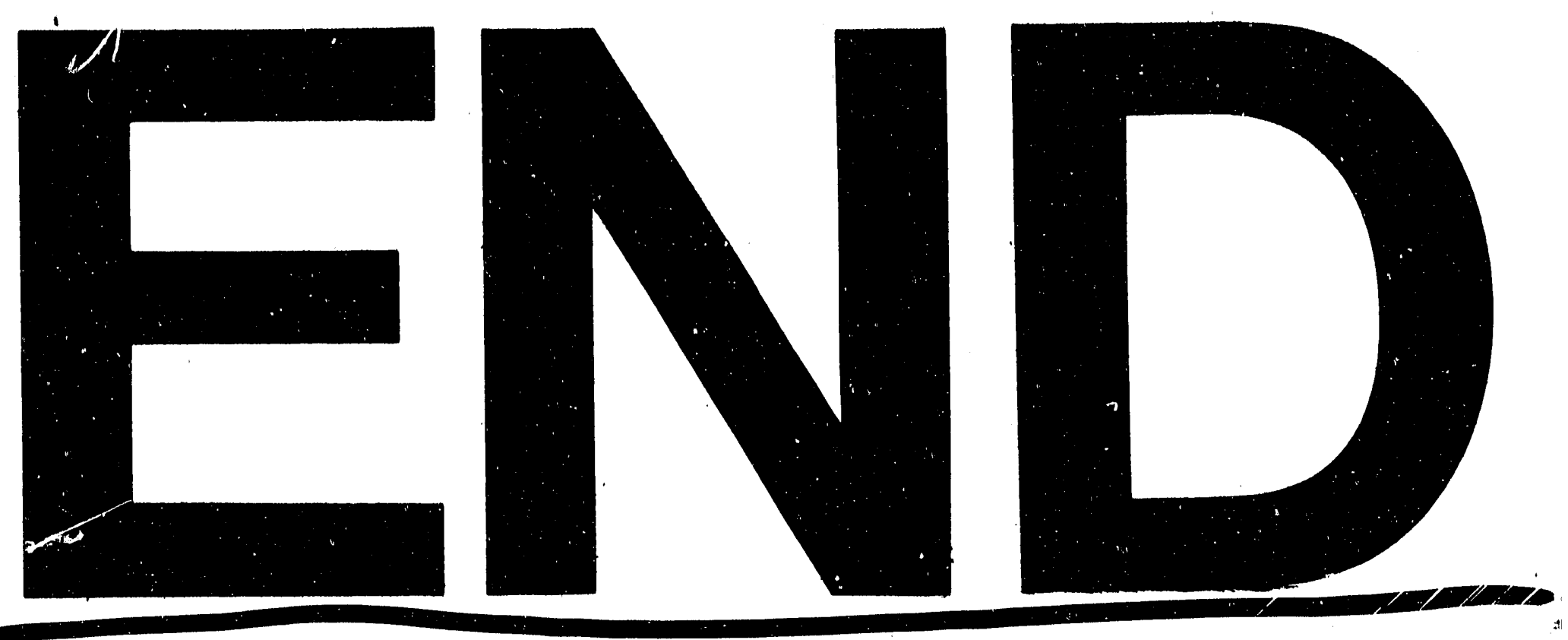

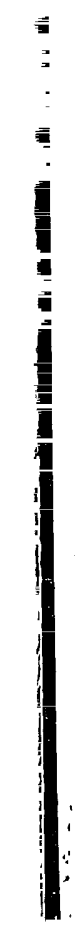
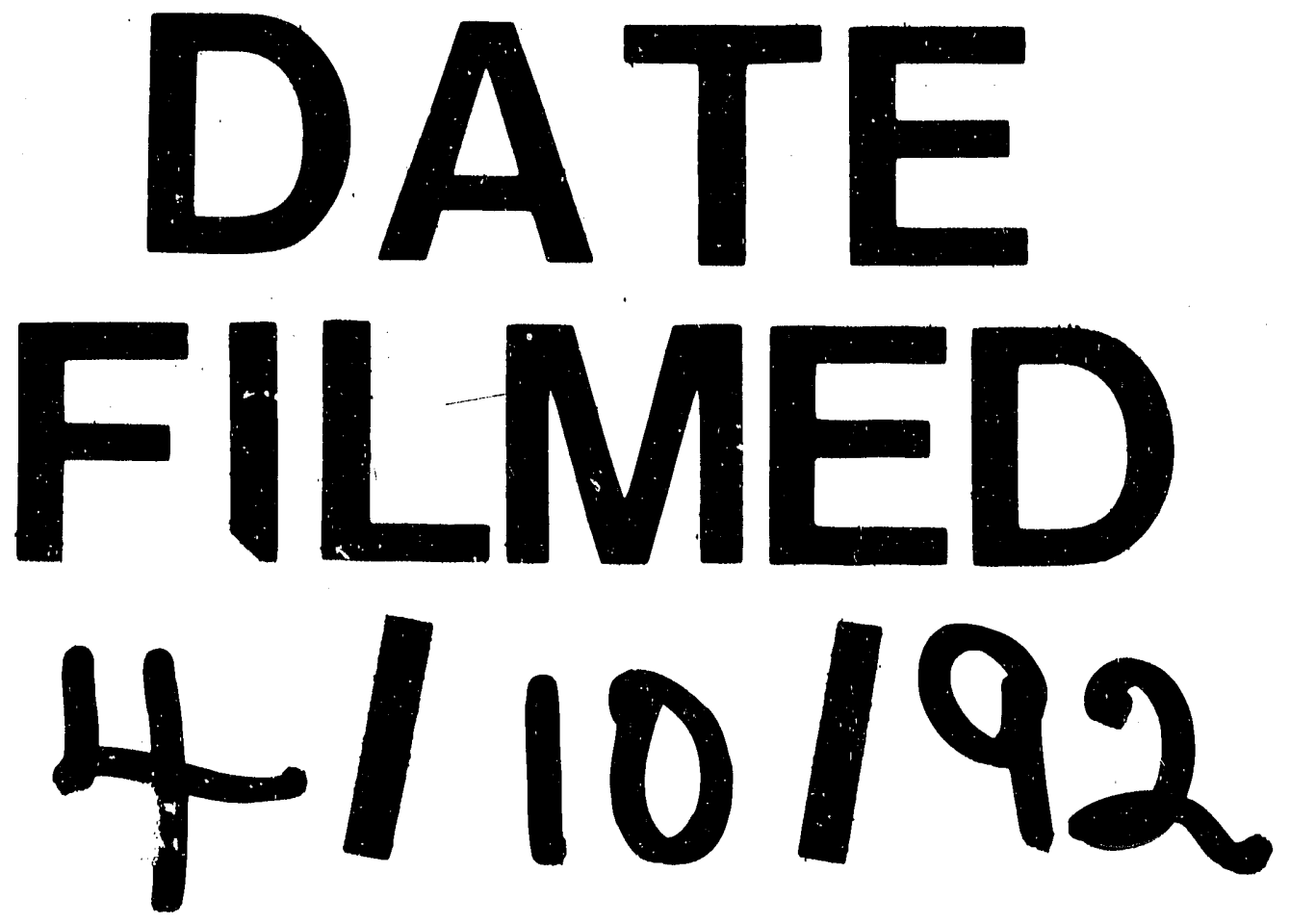
